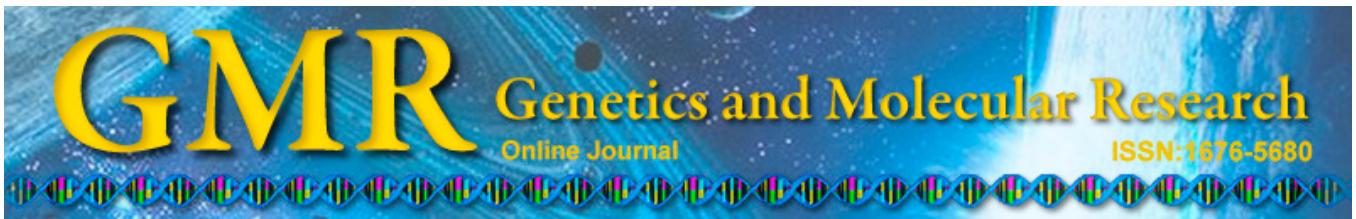

\title{
MTHFR A1298C polymorphism and ovarian cancer risk: a meta-analysis
}

\author{
X.Y. Shi ${ }^{1}$ and Q. Shen ${ }^{2}$ \\ ${ }^{1}$ Department of Clinical Laboratories, Hangzhou First People's Hospital, \\ Hangzhou, China \\ ${ }^{2}$ Department of Nosocomial Infection Management, \\ Hangzhou First People's Hospital, Hangzhou, China \\ Corresponding author: Q. Shen \\ E-mail: shenq1000@126.com
}

Genet. Mol. Res. 14 (3): 8211-8218 (2015)

Received September 4, 2014

Accepted March 18, 2015

Published July 27, 2015

DOI http://dx.doi.org/10.4238/2015.July.27.8

\begin{abstract}
Numerous studies have evaluated the association between the MTHFR A1298C polymorphism and ovarian cancer risk. However, the specific association is still controversial. Therefore, we performed the present meta-analysis. A systematic literature search of PubMed and Embase databases was undertaken in August 2014, and the reference lists of articles were retrieved. ORs with their $95 \% \mathrm{CI}$ were calculated to evaluate the strength of the association. Meta-analysis was performed using the STATA version 12.0 software package and publication bias was investigated by Begg's funnel plot. Five case-control studies from three publications (with 7026 subjects) on the relationship between the MTHFR A1298C polymorphism and ovarian cancer were analyzed by meta-analysis. Overall, no significant variation in ovarian cancer risk was detected in any of the genetic models (AA vs CC: $\mathrm{OR}=0.93,95 \% \mathrm{CI}=0.78-1.10$; AA vs AC: $\mathrm{OR}=1.02,95 \% \mathrm{CI}=0.92-1.13$; dominant model: $\mathrm{OR}=1.00,95 \% \mathrm{CI}=$ 0.91-1.10; recessive model: $\mathrm{OR}=0.92,95 \% \mathrm{CI}=0.78-1.08)$. In conclusion, this meta-analysis suggests that the A1298C polymorphism in the MTHFR gene may be not associated with susceptibility to ovarian cancer.
\end{abstract}

Key words: A1298C polymorphism; MTHFR gene; Ovarian cancer 


\section{INTRODUCTION}

Ovarian cancer is the most common cause of death from gynecological malignancies because it is usually diagnosed at an advanced stage, and the 5-year survival rate is often below $25-30 \%$ (Hanna and Adams, 2006). In the early stages, women are generally asymptomatic or have non-specific symptoms, making early-stage ovarian cancer difficult to diagnose (Asadollahi et al., 2010). The disease is often sporadic; in 2009, the American Cancer Society estimated that 21,550 women would be diagnosed with ovarian cancer (An et al., 2010). Despite the public health importance of ovarian cancer, its etiology remains unclear (Pennington and Swisher, 2012). Evidence suggests that family history, infertility, and age are risk factors for ovarian cancer, while increased parity, oral contraceptive use, hysterectomy, and tubal ligation are protective factors (Collaborative Group on Epidemiological Studies of Ovarian Cancer, et al., 2008). In addition, many studies suggest that genetic factors play an important role in the etiology of ovarian cancer. Germline mutations in the high penetrance genes BRCA1 and BRCA2 have been reported to be associated with ovarian cancer (Gayther et al., 1999).

Folate is indispensably required for DNA synthesis, and the methylation of DNA and histones. Folate deficiency (at low normal levels), due to DNA damage resulting from impaired nucleotide excision repair, is associated with risk of cancer in humans (Navarro Silvera et al., 2006). The 5,10-methylenetetrahydrofolate reductase (MTHFR) gene encodes a key limiting enzyme that controls the metabolism of folate and methionine, which are critical components required for nucleotide synthesis and DNA methylation, respectively (Frosst et al.,1995). The MTHFR gene comprises 11 exons and has a chromosomal locus of 1p36.3; it codes cDNA of $2.2 \mathrm{~kb}$ and produces a protein of 656 amino acids (Goyette et al., 1998). Several polymorphisms in the MTHFR gene have been identified. However, only C677T and A1298C polymorphisms have been expressed and confirmed as affecting enzyme activity (Frosst et al.,1995). The A1298C polymorphism is an A to C transition at base pair 1298 leading to a glutamate to alanine substitution. The MTHFR $1298 \mathrm{C}$ allele frequency is approximately $20-$ $70 \%$ in Asia, $24-46 \%$ in Europe, and 0-15\% in America (Yang et al., 2013).

A number of studies have been conducted to investigate the association between MTHFR A1298C polymorphism and ovarian cancer risk, but the results are somewhat controversial and underpowered. Meta-analysis can be a useful tool in detecting an association that could otherwise remain masked in the sample size studies, especially in those evaluating rare allele frequency polymorphisms (Attia et al., 2003). To derive a more precise estimation of the relationship between MTHFR A1298C polymorphism and ovarian cancer risk, we conducted a meta-analysis of all available case-control studies relating the $\mathrm{A} 1298 \mathrm{C}$ polymorphism of the MTHFR gene to the risk of ovarian cancer.

\section{MATERIAL AND METHODS}

\section{Selection of studies}

We performed an electronic search of the PubMed and Embase databases to retrieve papers available from August 2014 linking MTHFR A1298C polymorphism and susceptibility to ovarian cancer, using the following key words: "ovarian cancer" or "MTHFR"; and "A1298C", or "polymorphism", or "allele", or "genetic variant", or "variants". We did not set any restriction on the language of the published literature. All the searched studies were 
retrieved, and their references were checked for other relevant publications. If more than one study by the same author using the same case series was published, either the study with the largest sample size or the most recently published study was included.

\section{Inclusion and exclusion criteria}

To be included in the meta-analysis, studies had to satisfy the following inclusion criteria: a) case-control studies that addressed ovarian cancer cases and healthy controls; b) all patients diagnosed with ovarian cancer were confirmed by pathological or histological examinations; and c) published data about the frequencies of alleles or genotypes were sufficient. Studies were excluded when they were: a) not case-control studies that evaluated the association between MTHFR A1298C polymorphism and ovarian cancer risk; b) duplicates of previous publications; c) publications with incomplete data; or d) meta-analyses, letters, reviews, or editorial articles.

\section{Data extraction}

Data from the published studies were extracted independently by two authors and entered on a standardized form. For each study, the following characteristics and numbers were collected: the first author's name; the year of publication; the country of the study; ethnicity; the number of cases and controls; and the numbers of genotyped cases and controls. We did not require a minimum number of patients for a study to be included in our meta-analysis. In the case of disagreement, discrepancies of included studies were resolved by discussion.

\section{Statistical analysis}

The strengths of the associations between MTHFR A1298C polymorphism and susceptibility to ovarian cancer, estimated by OR and $95 \% \mathrm{CI}$ under a co-dominant model (AA vs $\mathrm{CC}$, AA vs $\mathrm{AC})$, a dominant model (CC+AC vs $\mathrm{AA}$ ), and a recessive model (AA+AC vs $\mathrm{CC}$ ), were calculated by the fixed-effect model or random-effect model. Statistical heterogeneity was assessed using the $\mathrm{I}^{2}$ test with a range between 0 and $100 \%$; $\mathrm{I}^{2}$ represents the variability that can be attributed to heterogeneity rather than chance. $\mathrm{I}^{2}$ values of 25,50 , and $75 \%$ were defined as low, moderate, and high estimates, respectively. When $\mathrm{I}^{2}>50 \%$ indicated heterogeneity across studies, the random-effect model was used for meta-analysis, otherwise the fixed-effect model was used. The distribution of the genotypes in the control population was tested for Hardy-Weinberg equilibrium using a goodness-of-fit chi-square test. Sensitivity analysis was conducted by switching the effect models. Publication bias was investigated by Begg's funnel plot, and $\mathrm{P}<0.05$ was considered to be statistically significant publication bias. All analyses were performed using STATA version 12.0 (StataCorp, College Station, TX, USA), and the significance level was set at 0.05. To ensure the reliability and accuracy of the results, two authors entered the data into the statistical software programs independently with the same results.

\section{RESULTS}

\section{Search results}

The searches initially retrieved 21 studies. First, the abstracts were read and studies 
other than randomized controlled trials, duplicate publications, and studies that did not meet the inclusion criteria were excluded. Finally, five case-control studies from three publications were included in this meta-analysis (Terry et al., 2010; Webb et al., 2011; Song et al., 2012). The flow chart for the study selection is summarized in Figure 1. The five case-control studies selected included a total of 3480 cases and 3546 healthy controls. The publication years of the studies considered ranged from 2000 to 2014. All the articles were written in English and based on healthy people. The distribution of genotypes in the controls was consistent with the Hardy-Weinberg equilibrium in all studies. Of the five studies, three used TaqMan probe, one used a polymerase chain reaction-restriction fragment length polymorphism probe, and one used the MassARRAY method. The main characteristics of eligible studies are summarized in Table 1.

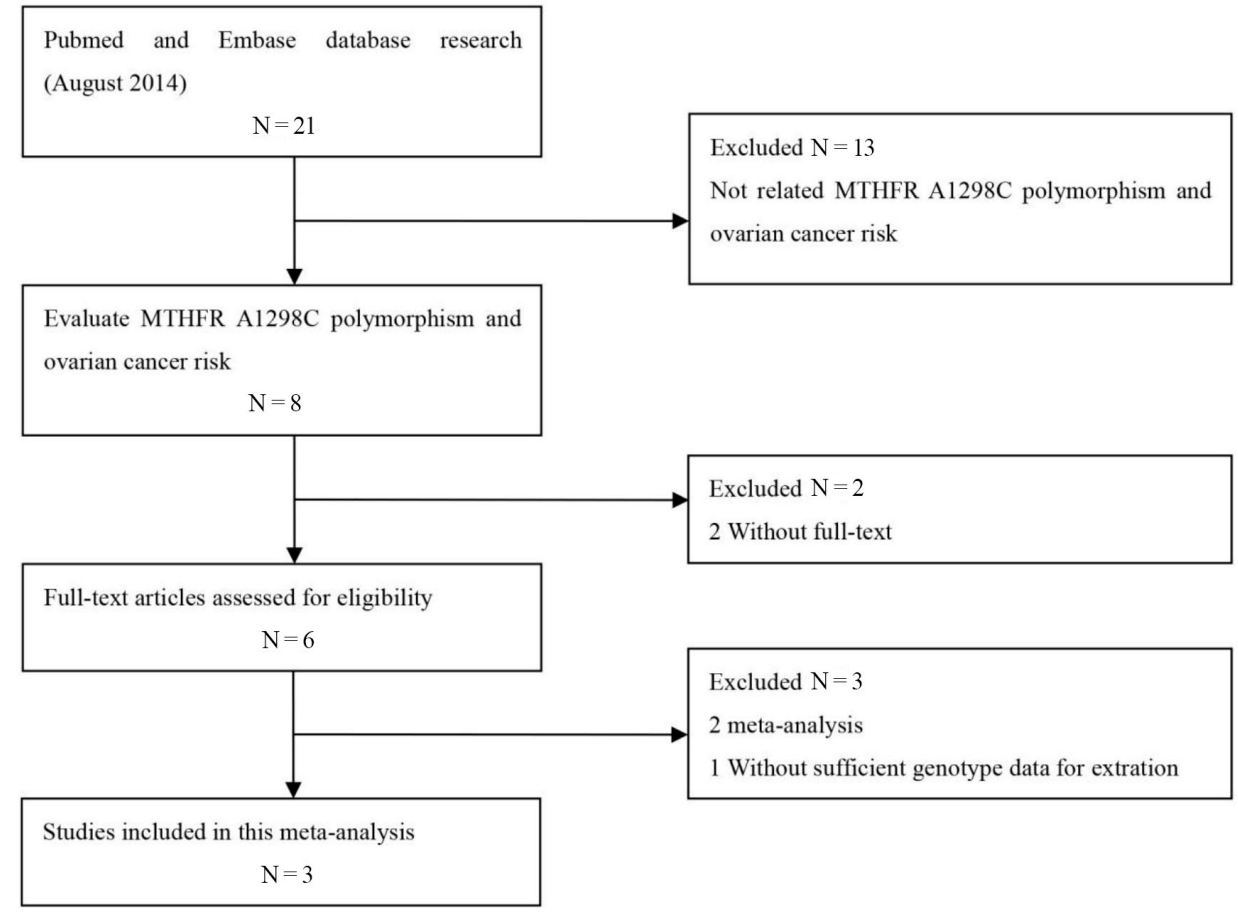

Figure 1. Flow diagram of study searching and selection process.

Table 1. Characteristics of the studies included for meta-analysis.

\begin{tabular}{|c|c|c|c|c|c|c|c|c|c|c|c|}
\hline \multirow[t]{2}{*}{ Study included } & \multirow[t]{2}{*}{ Year } & \multirow[t]{2}{*}{ Area } & \multirow[t]{2}{*}{ Cases } & \multirow[t]{2}{*}{ Controls } & \multicolumn{3}{|c|}{ Genotypes for cases } & \multicolumn{3}{|c|}{ Genotypes for controls } & \multirow[t]{2}{*}{ HWE test } \\
\hline & & & & & $\mathrm{AA}$ & $\mathrm{AC}$ & $\mathrm{CC}$ & $\mathrm{AA}$ & $\mathrm{AC}$ & $\mathrm{CC}$ & \\
\hline Terry et al. (NEC) & 2010 & America & 1120 & 1160 & 515 & 430 & 93 & 534 & 450 & 109 & 0.32 \\
\hline Terry et al. (NHS) & 2010 & America & 158 & 496 & 68 & 67 & 18 & 236 & 200 & 48 & 0.56 \\
\hline Terry et al. (MAY) & 2010 & America & 364 & 412 & 173 & 149 & 42 & 189 & 180 & 43 & 0.99 \\
\hline Webb et al. & 2011 & Australian & 1638 & 1278 & 770 & 693 & 175 & 598 & 561 & 119 & 0.44 \\
\hline Song et al. & 2012 & China & 200 & 200 & 107 & 77 & 16 & 112 & 79 & 9 & 0.29 \\
\hline
\end{tabular}

NEC: New England-Based Case-Control Study; NHS: Nurses' Health Study; MAY: Mayo Clinic Ovarian Cancer Case Control Study. 


\section{Quantitative data synthesis}

A summary of the meta-analysis findings of the association between MTHFR A1298C polymorphism and ovarian cancer risk is shown in Table 2 and Figure 2. We did not find significant association between MTHFR A1298C polymorphism and ovarian cancer risk in any of the genetic models (AA $v s \mathrm{CC}$ : $\mathrm{OR}=0.93,95 \% \mathrm{CI}=0.78-1.10$; $\mathrm{AA} v s \mathrm{AC}$ : $\mathrm{OR}=1.02$, $95 \% \mathrm{CI}=0.92-1.13$; the dominant model: $\mathrm{OR}=1.00,95 \% \mathrm{CI}=0.91-1.10$; the recessive model: $\mathrm{OR}=0.92,95 \% \mathrm{CI}=0.78-1.08)$. Sensitivity analysis was performed by comparing the results of fixed-and random-effect models and the results were not altered, suggesting that the data in this meta-analysis were relatively stable and credible.

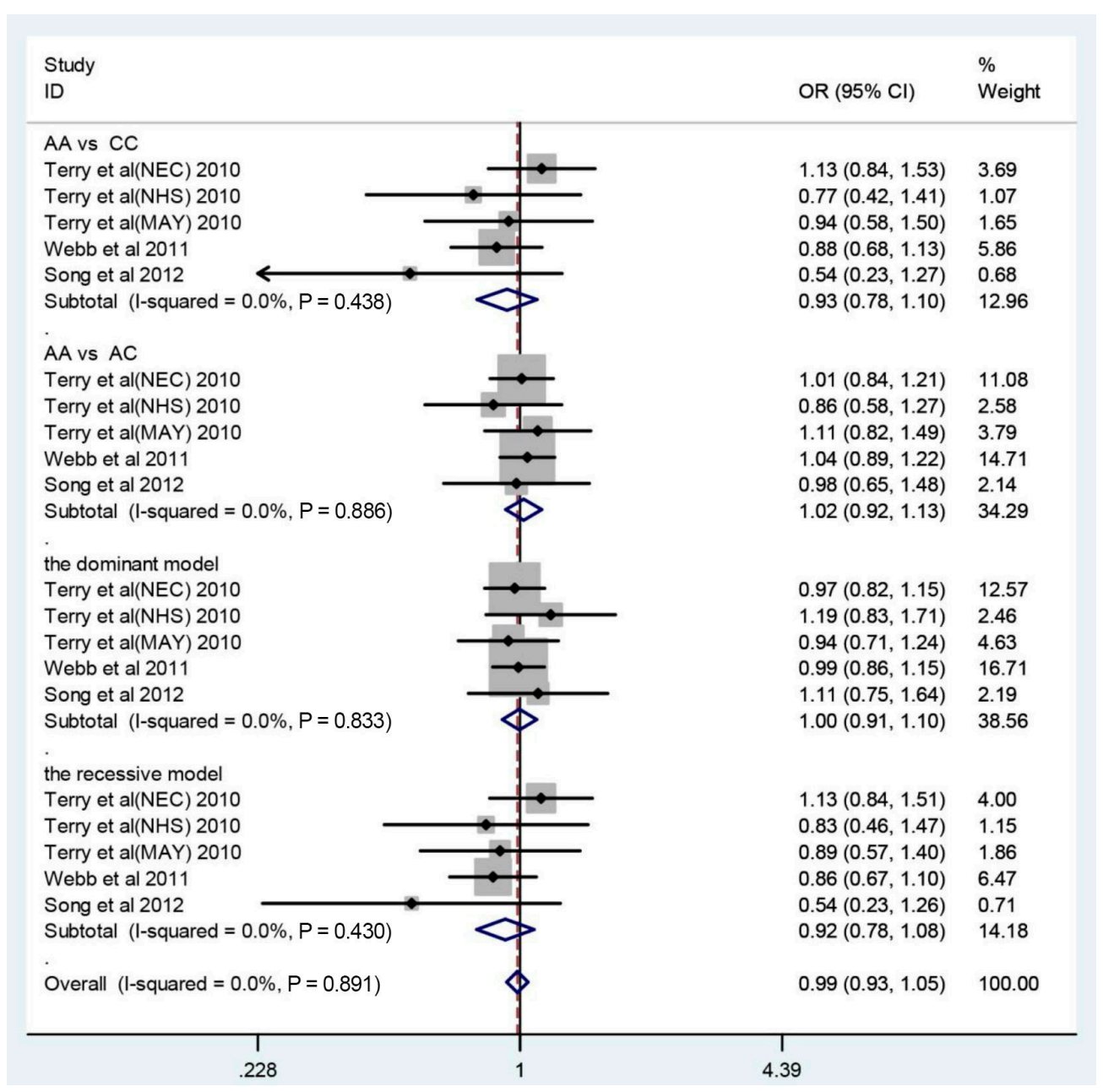

Figure 2. Meta-analysis of the relationship between the MTHFR A1298C polymorphism and ovarian cancer risk. The squares and horizontal lines correspond to the study-specific odds ratios (OR) and 95\% confidence intervals (CI). 
Table 2. Summary ORs and $95 \% \mathrm{CI}$ of the studies included for meta-analysis.

\begin{tabular}{|c|c|c|c|c|c|c|c|c|c|c|}
\hline \multirow[t]{2}{*}{ Subgroup } & \multirow[t]{2}{*}{ Genetic model } & \multicolumn{2}{|c|}{ Sample size } & \multirow[t]{2}{*}{ Type of model } & \multicolumn{2}{|c|}{ Test of heterogeneity } & \multicolumn{2}{|c|}{ Test of association } & \multicolumn{2}{|c|}{ Test of publication bias } \\
\hline & & Case & Control & & $\mathrm{I}^{2}$ & $\mathrm{P}$ & OR & $95 \% \mathrm{CI}$ & $\mathrm{z}$ & $\mathrm{P}$ \\
\hline \multirow[t]{4}{*}{ Overall } & $\mathrm{AA} v s \mathrm{CC}$ & 3480 & 3546 & Fixed & $0.0 \%$ & 0.44 & 0.93 & $0.78-1.10$ & 0.24 & 0.81 \\
\hline & $\mathrm{AA} v s \mathrm{AC}$ & & & Fixed & $0.0 \%$ & 0.89 & 1.02 & $0.92-1.13$ & 0.24 & 0.81 \\
\hline & Dominant model & & & Fixed & $0.0 \%$ & 0.83 & 1.00 & $0.91-1.10$ & 0.24 & 0.81 \\
\hline & Recessive model & & & Fixed & $0.0 \%$ & 0.43 & 0.92 & $0.78-1.08$ & 0.24 & 0.81 \\
\hline
\end{tabular}

\section{Publication bias}

A Begg's funnel plot was created to assess the publication bias of the literature. The shape of the funnel plot did not reveal any obvious evidence of asymmetry (Table 2 and Figure 3 ), indicating that our results were statistically robust.

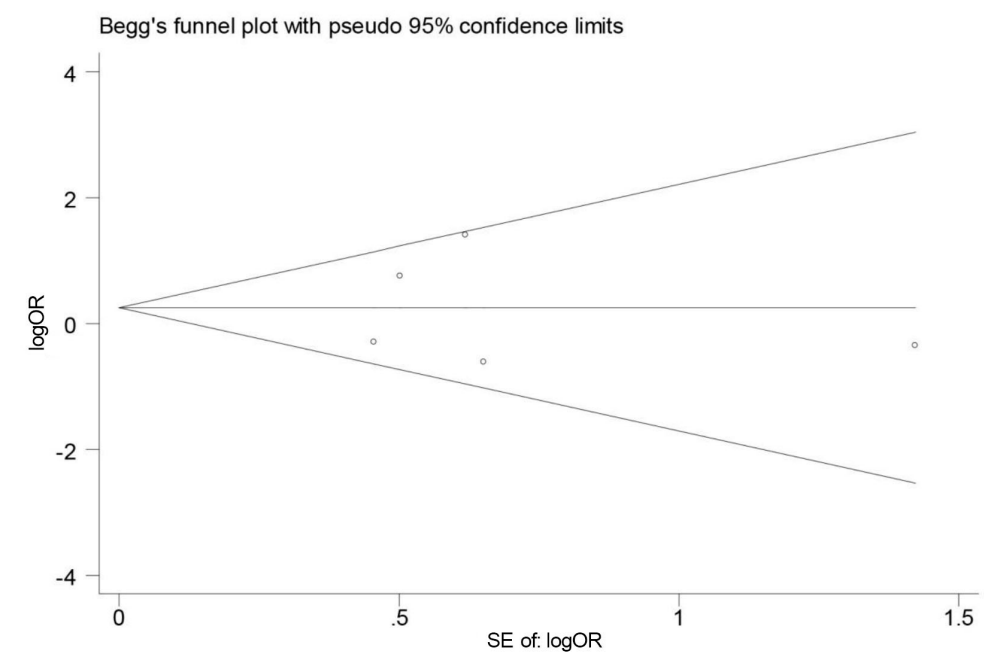

Figure 3. Begg funnel plot test of publication bias for the association between the MTHFR A1298C polymorphism and ovarian cancer risk.

\section{DISCUSSION}

The folate metabolism pathway plays an important role in DNA synthesis and DNA methylation, and it is directed by purine and pyrimidine synthesis. Epidemiological studies have shown an effective association between low folate intake and an increased cancer risk. MTHFR plays a vital role in the metabolism of folates. It has been reported that homozygotes (CC) for A1298C have only $60 \%$ of the normal enzyme activity (Weisberg et al., 1998). Decreased MTHFR activity may lead to an alteration of normal intracellular distribution of folate substrates and result in tumor susceptibility (Bagley and Selhub, 1998). Recently, several studies have focused on the association between the MTHFR A1298C polymorphism and ovarian cancer, but the results are controversial. The most likely reason for the inconsistencies among these studies is that they are single case-control studies with small sample sizes. We conducted this meta-analysis to help clarify the inconsistent findings, using several recently published studies. 
Our meta-analysis quantitatively assessed the association between MTHFR A1298C polymorphism and ovarian cancer risk. Ultimately, five case-control studies were included and assessed, encompassing a total of 3480 ovarian cancer patients and 3546 healthy controls. The results of the present meta-analysis revealed that MTHFR A1298C polymorphism is not associated with increased or decreased risk of ovarian cancer in the overall population. Further sensitivity analysis confirmed a significant association between MTHFR A1298C polymorphism and ovarian cancer risk. There was no evidence of publication bias in this meta-analysis for fracture. In addition, no study on African subjects was included in this meta-analysis. Further investigation of African subjects may be needed. As the eligible study number was limited in the meta-analysis, these results still require further investigation.

The development of ovarian cancer is due to the joint effect of multiple genes and gene-environment interactions. The potential function of MTHFR A1298C polymorphism might be affected by gene-gene interactions. Previous meta-analysis has shown that MTHFR C677T polymorphism is associated with ovarian cancer risk (Ding et al., 2012), and MTHFR gene C677T and A1298C variants are in linkage disequilibrium (Cicek et al., 2004; Shen et al., 2005). The linkage disequilibrium of C677T and A1298C may synergistically increase the risk of ovarian cancer. Further studies on gene-gene and gene-environment interactions should be taken into consideration for assessment of fracture risk.

This meta-analysis had some limitations. First, only five studies were included in the meta-analysis; the sample size was relatively small and may not have provided sufficient statistical power. Therefore, more studies with a larger sample size are required to provide a more representative statistical analysis. Second, subgroup analyses according to age, ethnicity, histological types, and other elements were not performed owing to insufficient relevant data available from the primary studies. Third, only published studies were included in the metaanalysis. Therefore, we cannot exclude the possibility of publication bias, although the results of the statistical tests showed it to be unlikely.

In conclusion, our meta-analysis indicates that MTHFR A1298C polymorphism is not associated with the risk of ovarian cancer. Large-scale case-control and population-based association studies are warranted to validate the risk identified in the current meta-analysis and investigate the potential gene-gene and gene-environment interactions.

\section{Conflicts of interest}

The authors declare no conflict of interest.

\section{REFERENCES}

An J, Wei Q, Liu Z, Lu KH, et al. (2010). Messenger RNA expression and methylation of candidate tumor-suppressor genes and risk of ovarian cancer - a case-control analysis. Int. J. Mol. Epidemiol. Genet. 1: 1-10.

Asadollahi R, Hyde CA and Zhong XY (2010). Epigenetics of ovarian cancer: from the lab to the clinic Epigenetics of ovarian cancer: from the lab to the clinic. Gynecol. Oncol. 118: 81-87.

Attia J, Thakkinstian A and D'Este C (2003). Meta-analyses of molecular association studies: methodologic lessons for genetic epidemiology. J. Clin. Epidemiol. 56: 297-303.

Bagley PJ and Selhub J (1998). A common mutation in the methylenetetrahydrofolate reductase gene is associated with an accumulation of formylated tetrahydrofolates in red blood cells. Proc. Natl. Acad. Sci. U S A. 95: 13217-13220.

Cicek MS, Nock NL, Li L, Conti DV, et al. (2004). Relationship between methylenetetrahydrofolate reductase C677T and A1298C genotypes and haplotypes and prostate cancer risk and aggressiveness. Cancer Epidemiol. Biomarkers Prev. 13: 1331-1336. 
Collaborative Group on Epidemiological Studies of Ovarian Cancer, Beral V, Doll R, Hermon C, et al. (2008). Ovarian cancer and oral contraceptives: collaborative reanalysis of data from 45 epidemiological studies including 23,257 women with ovarian cancer and 87,303 controls. Lancet 371: 303-314.

Ding XP, Feng L and Ma L (2012). MTHFR C677T polymorphism and ovarian cancer risk: a meta-analysis. Asian Pac. J. Cancer Prev. 13: 3937-3942.

Frosst P, Blom HJ, Milos R, Goyette P, et al. (1995). A candidate genetic risk factor for vascular disease: a common mutation in methylenetetrahydrofolate reductase. Nat. Genet. 10: 111-113.

Gayther SA, Russell P, Harrington P, Antoniou AC, et al (1999). The contribution of germline BRCA1 and BRCA2 mutations to familial ovarian cancer: no evidence for other ovarian cancer-susceptibility genes. Am. J. Hum. Genet. 65: 1021-1029.

Goyette P, Pai A, Milos R, Frosst P, et al. (1998). Gene structure of human and mouse methylenetetrahydrofolate reductase (MTHFR). Mamm. Genome 9: 652-656.

Hanna L and Adams M (2006). Prevention of ovarian cancer. Best. Pract. Res. Clin. Obstet. Gynaecol. 20: 339-362.

Navarro Silvera SA, Jain M, Howe GR, Miller AB, et al. (2006). Dietary folate consumption and risk of ovarian cancer: a prospective cohort study. Eur. J. Cancer Prev. 15: 511-515.

Pennington KP and Swisher EM (2012). Hereditary ovarian cancer: beyond the usual suspects. Gynecol. Oncol. 124: 347-353.

Shen H, Newmann AS, Hu Z, Zhang Z, et al. (2005). Methylenetetrahydrofolate reductase polymorphisms/haplotypes and risk of gastric cancer: a case-control analysis in China. Oncol. Rep. 13: 355-360.

Song CX, Ping L and Ting W (2012). Folate, MTHFR C677T and A1298C polymorphisms with the relationship with ovarian cancer risk among Chinese females. Afr. J. Microbiol. Res. 6: 4761-4766.

Terry KL, Tworoger SS, Goode EL, Gates MA, et al. (2010). MTHFR polymorphisms in relation to ovarian cancer risk. Gynecol. Oncol. 119: 319-324.

Webb PM, Ibiebele TI, Hughes MC, Beesley J, et al. (2011). Folate and related micronutrients, folate-metabolising genes and risk of ovarian cancer. Eur. J. Clin. Nutr. 65: 1133-1140.

Weisberg I, Tran P, Christensen B, Sibani S, et al. (1998). A second genetic polymorphism in methylenetetrahydrofolate reductase (MTHFR) associated with decreased enzyme activity. Mol. Genet. Metab. 64: 169-172.

Yang B, Liu Y, Li Y, Fan S, et al. (2013). Geographical distribution of MTHFR C677T, A1298C and MTRR A66G gene polymorphisms in China: findings from 15357 adults of Han nationality. PLoS One 8: e57917. 